\title{
Stage II Mycosis Fungoides and Sezary Syndrome AJCC v8
}

National Cancer Institute

\section{Source}

National Cancer Institute. Stage /I Mycosis Fungoides and Sezary Syndrome A/CC V8. NCI

Thesaurus. Code C141350.

Stage II includes: IIA (T1,2, N1,2, M0, B0,1); IIB (T3, N0-2, M0, B0,1). T1: Limited patches, papules, and/or plaques covering less than $10 \%$ of the skin surface. T2: Patches, papules, or plaques covering $10 \%$ or more of the skin surface. T3: One or more tumors (equal or greater than $1 \mathrm{~cm}$ in diameter). N0: No clinically abnormal peripheral lymph nodes; biopsy not required. N1: Clinically abnormal peripheral lymph nodes; histopathology Dutch grade 1 or National Cancer Institute (NCl) LN0-2. N2: Clinically abnormal peripheral lymph nodes; histopathology Dutch grade 2 or NCI LN3. M0: No visceral organ involvement. B0: Absence of significant blood involvement: $5 \%$ or less of peripheral blood lymphocytes are atypical (Sezary cells). B1: Low blood tumor burden: more than $5 \%$ of peripheral blood lymphocytes are atypical (Sezary cells) but does not meet the criteria of B2. (AJCC 8th ed.) 